\title{
Doit-on opposer approches françaises et allemandes de la reconnaissance?
}

Should we Distinguish between French and German Approaches to Recognition?

\section{Emmanuel Renault}

\section{(2) OpenEdition}

\section{Journals}

Édition électronique

URL : https://journals.openedition.org/cps/5064

DOI : $10.4000 /$ cps.5064

ISSN : 2648-6334

\section{Éditeur}

Presses universitaires de Strasbourg

\section{Édition imprimée}

Date de publication : 12 décembre 2021

Pagination : 131-146

ISBN : 979-10-344-0098-0

ISSN : $1254-5740$

Référence électronique

Emmanuel Renault, " Doit-on opposer approches françaises et allemandes de la reconnaissance? », Les Cahiers philosophiques de Strasbourg [En ligne], 50 | 2021, mis en ligne le 12 décembre 2021. consulté le 09 novembre 2022. URL : http://journals.openedition.org/cps/5064 ; DOI : https://doi.org/ $10.4000 /$ cps.5064

\section{(c) (1) (ㅇ)}

Creative Commons - Attribution - Pas d'Utilisation Commerciale - Partage dans les Mêmes Conditions 4.0 International - CC BY-NC-SA 4.0

https://creativecommons.org/licenses/by-nc-sa/4.0/ 


\section{Doit-on opposer approches françaises et allemandes de la reconnaissance?}

Emmanuel Renault

La thèse d'une forte spécificité de la pensée française d'après-guerre, ou de manière plus restrictive de celle des années 1960, par rapport à la philosophie allemande, jouit aujourd'hui d'une forme d'évidence rarement interrogée, ce qui est en fait très curieux dans la mesure où l'on aurait pu penser qu'aujourd'hui, à l'époque de la mondialisation accomplie de la philosophie, le nationalisme épistémologique a cessé de constituer une option tenable. Cette évidence non interrogée s'exprime notamment dans la manière dont sont comparées les approches philosophiques de la reconnaissance en France et en Allemagne. On rencontre à ce propos deux types de jugements distincts, plus souvent implicites que vraiment formulés, et quand ils le sont, plus souvent formulés sous le mode du "ça va de soi» que vraiment argumentés: le premier est que l'idée de théorie de la reconnaissance est allemande, au sens où l'on ne trouverait pas chez les auteurs français ce qui pourrait à bon droit être désigné comme une théorie de la reconnaissance; le second argument affirme que le propre des approches philosophiques françaises de la reconnaissance est qu'elles saisissent la face négative de la reconnaissance, sa dimension conflictuelle ou aliénante, alors que les Allemands rattacheraient la reconnaissance à l'autonomie individuelle et collective et à la réconciliation ${ }^{1}$. Dans ce qui suit, on considérera

1 Une troisième manière d'interpréter la spécificité française se fonderait sur l'idée, développée par Butler dans Sujets du désir. Réflexions hégéliennes en France au XX siècle, suivant laquelle la philosophie française, chez Kojève, Hyppolite, Sartre, Derrida, Lacan, Deleuze et Foucault, exploiterait de différentes manières la conception hégélienne du désir. Je ne discuterai pas cette manière de spécifier la philosophie française d'après-guerre, d'une 
successivement ces deux arguments avant de poser le problème des traditions nationales dans la philosophie contemporaine.

\section{La promotion philosophique du concept de reconnaissance est-elle une opération allemande?}

Telle est l'idée reçue. Et pourtant, jusqu'à une date assez récente, il n'y avait qu'en France qu'un rôle décisif avait été conféré au concept de reconnaissance. Alexandre Kojève a fait de ce concept le maître mot de la philosophie hégélienne, ce qui ne semble être jamais venu à l'esprit de personne dans l'histoire de l'hégélianisme avant lui. Il est probablement responsable du fait qu'ensuite, Jean-Paul Sartre, Jacques Lacan, Simone de Beauvoir et Frantz Fanon, notamment ${ }^{2}$, se sont emparés de ce concept pour formuler certaines de leurs thèses principales. Dans la période d'après-guerre, et jusqu'à La lutte pour la reconnaissance d'Axel Honneth ${ }^{3}$, c'est-à-dire 1991, aucun philosophe allemand n'avait donné autant d'importance à l'idée de reconnaissance. Dans la période qui court des années 1930 aux années 1990, la théorisation philosophique de la reconnaissance apparaît donc comme un phénomène principalement français.

Certes, si l'on entend par «théorie de la reconnaissance» une construction théorique systématique prenant la reconnaissance pour thème principal, on peut bien dire qu'on ne trouve pas de théorie de la reconnaissance chez Alexandre Kojève, Jean-Paul Sartre, Jacques Lacan, Simone de Beauvoir et Frantz Fanon, mais on n'en trouve pas non plus chez Hegel lui-même! Chez ce dernier, en effet, le concept de reconnaissance se voit bien conférer une importance fondamentale dans la mesure où il désigne l'imbrication de l'individuel et du collectif qui caractérise l'esprit en tant qu'esprit objectif: «un Je qui est un Nous et

part parce qu'elle s'applique à une série d'auteurs chez qui la question de la reconnaissance n'est pas discutée, ou très marginalement (Derrida, Deleuze et Foucault), d'autre part parce qu'elle ne permet pas de rendre compte de la manière dont des auteurs aussi importants que Beauvoir et Fanon ont abordé la question de la reconnaissance.

2 On peut ajouter Merleau-Ponty à la liste comme le souligne Jean-Philippe Deranty dans l'entrée consacrée à cet auteur dans L. Siep, H. IKÄHEIMO, M. QuAnTe (hrsg.), Handbuch Anerkennung.

3 Cf. A. Honneth, La Lutte pour la reconnaissance. 
un Nous qui est un Je $»^{4}$, mais ce concept fait l'objet d'un traitement dispersé, non systématisé, dans la Phénoménologie et dans d'autres parties du système $e^{5}$.

En Allemagne, l'intérêt porté à la reconnaissance est relativement tardif. L'impulsion vient probablement de l'article "Travail et interaction. Remarques sur la Philosophie de l'esprit de Hegel à Iéna" publié par Jürgen Habermas une première fois en 1967 avant d'être reproduit dans le volume La Technique et la Science comme "idéologie» en $1968^{6}$. Il s'agissait pour Jürgen Habermas de chercher dans la distinction entre travail et reconnaissance, élaborée dans les philosophies de l'esprit d'Iéna, le moyen de critiquer le primat marxiste de la production tout en opposant l'agir communicationnel à l'agir instrumental. Il est frappant que Jürgen Habermas ait ensuite élaboré la distinction de ces deux formes de l'agir social sans plus de référence à la reconnaissance. Dans l'itinéraire habermassien, l'intérêt pour la reconnaissance est transitoire et principalement historiographique: Habermas s'est en fait contenté de chercher une distinction conceptuelle, celle du travail et de la reconnaissance, chez le Hegel d'Iéna, sans pour autant juger que le thème de la reconnaissance était philosophiquement décisif'. Il est d'ailleurs significatif que cet article ait produit ses effets les plus notables tout d'abord dans le domaine de la Hegel-Forschung. Ludwig Siep et Andreas Wildt ${ }^{8}$ ont engagé des recherches sur les conceptions hégéliennes de la reconnaissance à l'époque d'Iéna, au cours des années 1970 et 1980, dont les enjeux étaient principalement internes aux études hégéliennes. Ces recherches ont ensuite donné à Axel Honneth l'idée d'une actualisation des conceptions hégéliennes de la reconnaissance, telles qu'elles avaient été élaborées à l'époque d'Iéna, comme en témoignent notamment les références à ces deux auteurs dans La Lutte pour la reconnaissance.

4 Cf. G. W. F. Heger, Phénoménologie de l'esprit, p. 262, trad. modifiée. Sur le sens de cette formule, voir F. Fischbach, "L'agir de tous et de chacun": l'autre mot de l'esprit (chapitre 5, sections B et C)».

5 Voir à ce propos, F. Fischbach, Hegel, Fichte. La reconnaissance.

6 Cf. J. Habermas, La Technique et la Science comme «idéologie», p. 209-221.

$7 C f$. E. Renault, "Travail et interaction. Origine et implications d'une distinction".

8 Cf. L. Siep, Anerkennung als Prinzip der praktischen Philosophie: Untersuchungen zu Hegels Jenaer Philosophie des Geistes; A. WiLdt, Autonomie und Anerkennung. Hegels Moralitätskritik im Lichte seiner Fichte-Rezeption. 
Cela signifie-t-il que du point de vue des études hégéliennes au moins, la promotion de la reconnaissance en thème digne d'une attention savante s'est produite tout d'abord en Allemagne, et ensuite seulement en France où le rapport des théorisations de la reconnaissance à Hegel serait longtemps resté celui d'Alexandre Kojève, à savoir celui de l'interprétation libre orientée par des enjeux philosophiques contemporains? De nouveau, cette thèse est intenable. C'est bien de France que sont venues les premières études philologiquement informées du concept hégélien de reconnaissance. Elles étaient précisément destinées à récuser, dans un rapport polémique à Alexandre Kojève, l'idée que Hegel aurait donné une importance fondamentale à la reconnaissance et à la lutte pour la reconnaissance. On doit cette clarification rigoureuse à Pierre-Jean Labarrière, dès son premier ouvrage: Structures et mouvement dialectique dans la Phénoménologie de l'esprit de Hegel, datant de $1968^{\circ}$. C'est le fait que la philosophie française d'après-guerre ait donné beaucoup plus d'importance aux conceptions hégéliennes de la reconnaissance que la philosophie allemande à la même époque, tout en s'appuyant sur des lectures de Hegel très discutables, qui a conduit des spécialistes de Hegel, en France plus tôt qu'en Allemagne, à faire de la reconnaissance l'objet d'une attention philologique rigoureuse.

De tout cela, il semble nécessaire de conclure que contrairement aux préjugés, l'idée de reconnaissance n'est pas moins philosophiquement française qu'allemande: avant Honneth, aucun Allemand (pas même Habermas) n'avait eu l'idée d'associer des enjeux philosophiques fondamentaux au thème de la reconnaissance (comme chez Sartre, Beauvoir et Fanon notamment), et même dans le domaine des études hégéliennes, les Français ont précédé les Allemands, précisément parce qu'ils voulaient lutter contre la trop grande importance conférée par Kojève à l'idée de lutte pour la reconnaissance.

9 Cf. P.-J. LABARrière, Structures et mouvement dialectique dans la Phénoménologie de l'esprit de Hegel, puis G. Jarczyk, P. J. Labarrière, Les Premiers Combats de la reconnaissance. Voir également, pour une polémique plus soutenue avec Alexandre Kojève: G. JarczyK, P.-J. Labarrière, De Kojève à Hegel. 150 ans de pensée hégélienne en France. 
DOIT-ON OPPOSER APPROCHES FRANÇAISES ET ALLEMANDES DE LA RECONNAISSANCE?

\section{Conflit et aliénation versus émancipation et réconciliation?}

Faut-il opposer approches françaises de la reconnaissance comme conflit et aliénation, et approches allemandes de la reconnaissance comme autonomie et réconciliation? Cette opposition semble plausible, et elle trouve une formulation argumentée dans le dernier ouvrage d'Axel Honneth, La Reconnaissance. Histoire européenne d'une idée ${ }^{10}$. Le thème de la lutte pour la reconnaissance, et donc la dimension agonistique de la reconnaissance, sont fondamentaux chez Alexandre Kojève, qui établit à tort (philologiquement) un lien essentiel entre reconnaissance et lutte pour la reconnaissance chez Hegel ${ }^{11}$. On retrouve un tel lien chez Simone de Beauvoir et Frantz Fanon ${ }^{12}$. En outre, en identifiant tendanciellement lutte pour la reconnaissance et dialectique du maître et de l'esclave, Alexandre Kojève a rendu possible une phénoménologie de l'aliénation par la reconnaissance que l'on trouve aussi bien chez Jean-Paul Sartre et Frantz Fanon que chez Jacques Lacan ${ }^{13}$. Que la reconnaissance ne puisse être qu'aliénante, voilà une thèse qu'on ne trouve certes pas chez Frantz Fanon, mais bien chez Jean-Paul Sartre et Jacques Lacan, et cette idée semble pouvoir être rapprochée de la thèse du caractère assujettissant de la reconnaissance qui est au cœur des conceptions althussérienne et butlerienne de la reconnaissance ${ }^{14}$. Chez Jacques Rancière également, chez qui l'idée de reconnaissance semble toujours entendue comme reconnaissance de l'identité, reconnaissance signifie assujettissement ${ }^{15}$.

$10 C f$. A. Honneth, La Reconnaissance. Histoire européenne d'une idée.

11 Chez Hegel, le conflit de la reconnaissance n'est que la forme la plus immédiate, et donc la plus défectueuse, de la recherche de la reconnaissance; voir à ce propos, E. Renault, Reconnaissance, conflit, domination, chap. 1.

12 La manière dont Fanon s'approprie Hegel est médiatisée par la lecture du chapitre IV-A de la Phénoménologie de l'esprit par Simone de Beauvoir, comme l'a montré Matthieu Renault dans «Le genre de la race: Fanon, lecteur de Beauvoir». Pour une discussion de l'actualité de la conception agonistique de la reconnaissance qui est propre à Fanon, voir H. BentouhamiMolino, Races, cultures, identités, chap. 3.

13 Sur le rapport de Lacan à Hegel, voir J. Butler, Sujets du désir, p. 225-246.

14 D'où l'opposition entre les conceptions butlerienne et honnethienne de la reconnaissance, étudiée notamment par Kim Sang ONG-VAN-Cung, «Reconnaissance et vulnérabilité. Honneth et Butler».

15 D'où également l'opposition à Honneth, qui a donné lieu au dialogue suivant: J.-P. Deranty, K. Genel (dir.), Reconnaissance ou mésentente? Un dialogue critique entre Jacques Rancière et Axel Honneth. 
Au contraire, les approches allemandes de la reconnaissance penseraient la reconnaissance comme une condition de l'autonomie, et elles penseraient l'autonomie dans la perspective de la réconciliation des consciences de soi. Telle est l'idée largement partagée, à laquelle Axel Honneth donne une profondeur historique en faisant remonter les approches "françaises», qui soulignent de différentes manières la négativité de la reconnaissance, jusqu'à Rousseau et au contraste que sa critique de l'amour-propre forme avec la fondation kantienne de la moralité sur le respect de la personne humaine. Remarquons cependant que si l'on était remonté du côté français jusqu'aux matérialistes français ${ }^{16}$, voire à La Boétie ${ }^{17}$, on aurait abouti à d'autres conclusions. Par ailleurs, la spécificité des approches propres à la philosophie française d'après-guerre serait sans doute apparue sous un jour moins national s'il avait été davantage tenu compte du chaînon manquant entre la critique rousseauiste de l'amour-propre d'une part, et d'autre part la critique de la reconnaissance comme aliénation chez Jean-Paul Sartre, Jacques Lacan et Louis Althusser: l'interprétation libre par un émigré russe (Alexandre Kojève) d'un philosophe allemand (Hegel) à partir de thèmes puisés chez deux autres auteurs allemands (Marx et Heidegger).

Il est vrai que chez Hegel, du moins dans la Phénoménologie de l'esprit, la reconnaissance est la condition de l'autonomie, et que Hegel y affirme qu'il n'est pas possible d'obtenir de reconnaissance véritable par la lutte, mais seulement par la réconciliation des consciences de soi, telle qu'elle est thématisée à la fin du chapitre VI dans la dialectique du mal et de son pardon ${ }^{18}$. Tel serait le point de vue typiquement allemand que Jürgen Habermas aurait repris à son compte dans l'article "Travail et interaction", en faisait de la reconnaissance l'équivalent de l'agir communicationnel, et qu'Axel Honneth aurait ensuite systématisé en faisant des rapports de reconnaissance institutionnalisés le cœur de la liberté sociale. Cependant, chez Hegel, Habermas et Honneth, de même que chez d'autres auteurs également qui les relient les uns aux autres,

16 Cf. F. Toto, «L'impensé de Claude-Adrien Helvétius. Le problème de l'estime dans De l'esprit».

17 Cf. E. Renault, «Reconnaissance et domination chez La Boétie».

18 Voir à ce propos, C. Berner, «La critique de la conscience morale (chap. 6, section C)». 
comme Marx, la négativité de la reconnaissance est également un thème central.

Il semble difficile de contester que dans la Phénoménologie de l'esprit, la question de la reconnaissance est abordée à partir de celle du déni de reconnaissance qui peut conduire à la lutte pour la reconnaissance, et que cette lutte pour la reconnaissance peut n'avoir aucune issue satisfaisante compte tenu du fait que selon Hegel, la reconnaissance véritable n'est pas unilatérale et hiérarchique mais réciproque et égale. Il semble tout aussi difficile de contester qu'on trouve dans le chapitre IV-A de la Phénoménologie de l'esprit une analyse de la contribution de la reconnaissance à la domination ${ }^{19}$. Il en va de même chez Marx, auteur qui, curieusement, apparaît rarement dans les généalogies des approches allemandes de la reconnaissance. Qui plus est, dans ses écrits de jeunesse, ceux d'un jeune-hégélien encore pris dans ce qu'il appellera ensuite «l'idéologie allemande», Marx identifie la «reconnaissance réciproque», qui conditionne chez Hegel la véritable autonomie, à l'aliénation ${ }^{20}$. Serait-il plus Français qu'Allemand?

$\mathrm{Si}$ l'on considère maintenant la philosophie $\mathrm{du} \mathrm{xx}^{\mathrm{e}}$ siècle, les difficultés ne manquent pas non plus. Une première difficulté tient au fait que c'est en France, chez Alexandre Kojève et à sa suite, que l'on a pu croire en une réconciliation définitive des luttes de reconnaissance, non en Allemagne. Quoi qu'on puisse penser du rôle que Jürgen Habermas et Axel Honneth ont conféré à la conflictualité sociale, on doit porter à leur crédit de ne jamais avoir cru à la fin de l'histoire, contrairement à Alexandre Kojève, Francis Fukuyama ou Bernard Bourgeois. Une deuxième difficulté tient au fait que si Jürgen Habermas et Axel Honneth se sont tournés vers les Philosophies de l'esprit d'Iéna, c'est qu'ils ont repris à Adorno la thèse suivant laquelle la Phénoménologie de l'esprit portait la trace d'une métaphysique de l'esprit absolu, c'est-à-dire d'une métaphysique de la réconciliation du sujet et de l'objet et des sujets entre eux, qui la rendait inutilisable pour toute théorie sociale critique. Chez Axel Honneth tout particulièrement, du moins à l'époque de La Lutte

19 Voir à ce propos, E. Renault, Reconnaissance, conflit, domination, chap. 1.

20 Cf. K. Marx, "Notes sur James Mill», p. 30-31. Pour des transformations de la problématique hégélienne de la reconnaissance chez Marx, voir E. Renault, Reconnaissance, conflit, domination, chap. 2, et le dossier de la revue Ethical Theory and Practice, vol. 16, n 4, 2013. 
pour la reconnaissance, la reconnaissance est pensée à partir des esquisses de système d'Iéna, parce qu'il est considéré que Hegel y a accordé plus d'importance à la dimension conflictuelle de la reconnaissance que dans la Phénoménologie. Beau paradoxe: alors qu'Alexandre Kojève a fondé à partir de la Phénoménologie de l'esprit une théorie de la lutte pour la reconnaissance qui a influencé des théories françaises de la reconnaissance hostiles à l'exigence hégélienne de réconciliation, Jürgen Habermas et Axel Honneth, souvent jugés trop réconciliateurs, ont quant à eux insisté sur le fait que la perspective de la Phénoménologie était trop réconciliatrice pour être pertinente. Troisième difficulté, l'intention initiale d'Axel Honneth n'était pas seulement de valoriser le jeune Hegel, mais aussi de critiquer les tendances réconciliatrices dont était porteuse l'idée habermassienne d'agir communicationnel. Cette critique, il l'a développée dans un premier temps indépendamment de toute référence à la reconnaissance, dans sa thèse, Critique du pouvoir, publiée en $1985^{21}$. C'est sur Michel Foucault qu'il s'est alors appuyé. C'est seulement dans un deuxième temps qu'il a cherché à développer cette revalorisation de la conflictualité sous la forme d'une théorie de la reconnaissance, dans La Lutte pour la reconnaissance. Foucault n'y joue plus de rôle explicite, mais Honneth continue à se référer dans cet ouvrage à ce qu'il appelle une "tradition fragmentaire" en philosophie sociale, qui met le conflit au cœur du social. Les trois figures principales de cette tradition sont Marx, Sorel et Sartre - et Sartre discutant Fanon! Qu'il s'agisse de l'insistance sur la conflictualité en théorie sociale dans Critique $d u$ pouvoir, ou de la dimension conflictuelle de la reconnaissance dans La Lutte pour la reconnaissance, la prise en compte de la philosophie française d'après-guerre joue donc un rôle déterminant, ce qui brouille pour le moins l'opposition tranchée des Français soulignant la négativité de la reconnaissance et des Allemands pensant la reconnaissance comme réconciliée. Cette opposition est d'autant plus contestable qu'à l'époque de La Lutte pour la reconnaissance, Axel Honneth revendique le négativisme méthodologique d'Adorno ${ }^{22}$.

De nouveau, on pourrait d'ailleurs renverser les rôles et non pas seulement brouiller leur distribution. Car jusqu'à présent, nous avons

$21 C f$. A. Honneth, Critique du pouvoir.

22 Cf. E. Renault, "Théorie de la reconnaissance et négativisme méthodologique». 
fait comme si seul un philosophe allemand avait élaboré une théorie systématique de la reconnaissance, Axel Honneth en 1991, alors qu'il existe également une version française de cette entreprise: Parcours de la reconnaissance de Paul Ricoeur ${ }^{23}$. Or, ce dernier y a précisément reproché à Axel Honneth de donner trop d'importance à la dimension conflictuelle de la reconnaissance ${ }^{24}$ ! Il est douteux que La Lutte pour la reconnaissance soit un phénomène typiquement allemand, dans la mesure où elle met en dialogue Hegel non seulement avec des auteurs français mais aussi avec le pragmatisme américain - qui lui aussi hérite directement du concept hégélien de reconnaissance ${ }^{25}$. En revanche, Parcours de la reconnaissance est bien un phénomène typiquement français. Qui, hors de France, aurait pu forger le projet de développer une théorie philosophique à partir de l'analyse des différentes définitions du terme «reconnaissance» dans le Littré? Qui, hors de France, aurait pu soutenir que l'idée de gratitude, qui est exprimée par le terme «reconnaissance» et ses équivalents dans les langues latines, mais ni en allemand ni en anglais, doit être constitutive de toute pensée de la reconnaissance d'où la critique de Honneth, qui donne une vision trop conflictuelle de la reconnaissance en oubliant la reconnaissance comme gratitude? Remarquons en passant que le terme allemand "Anerkennung" ne véhicule pas non plus l'idée de re-cognition, que les termes latins, ainsi que l'équivalent anglais, véhiculent bien. Le projet d'un "parcours" de la reconnaissance dans ses trois sens de re-cognition, de reconnaissance réciproque et de gratitude est donc typiquement français par son objet, et il l'est également par sa méthode puisque cette dernière semble surtout faire sens selon les normes de la dissertation de philosophie générale, dont on sait qu'elle n'existe qu'en France.

L'opposition entre les Français conflictuels et les Allemands réconciliateurs est donc difficilement tenable. Lidée suivant laquelle les Allemands concevraient la reconnaissance comme condition de la liberté alors que les Français percevraient ses effets d'assujettissement ou d'aliénation n'est pas plus convaincante. Il est vrai que la reconnaissance

23 Cf. P. Ricoeur, Parcours de la reconnaissance. Trois études.

24 Sur cette critique, voir A. P. Olivier, «La reconnaissance comme lutte et la reconnaissance comme gratitude: un conflit d'interprétation entre Honneth et Ricœur".

25 Voir E. Renault, Reconnaissance, conflit, domination, chap. 3. 
reste conçue, de Hegel à Axel Honneth en passant par Jürgen Habermas, comme une condition de l'autonomie individuelle et collective. Mais cela n'interdit pas à Axel Honneth de souligner les effets de domination dont la reconnaissance positive peut être porteuse (par exemple dans l'article «La reconnaissance comme idéologie $\left.{ }^{26}\right)$, sans parler des différentes formes d'expériences sociales négatives et de réification dont le déni de reconnaissance est porteur (voir notamment le livre sur La Réification ${ }^{27}$ ). Par ailleurs, s'il est vrai qu'on peut considérer que la reconnaissance est fondamentalement aliénation pour Jean-Paul Sartre, Jacques Lacan et Louis Althusser, et qu'elle ne peut donc apporter aucune contribution à l'émancipation, il n'en va pas de même chez Frantz Fanon et Simone de Beauvoir, qui soulignent que l'émancipation ne peut être octroyée mais doit être obtenue par la lutte, et qui conçoivent cette lutte comme une lutte contre la non-reconnaissance de la pleine humanité des femmes et des racisés, en s'appuyant sur Hegel contre Sartre ${ }^{28}$. Seraient-ils moins Français, ou plus Allemands, que Jean-Paul Sartre, Jacques Lacan et Louis Althusser?

Dans les disqualifications du concept de reconnaissance que l'on entend de ce côté-ci du Rhin, on affirme fréquemment que la valorisation de la reconnaissance s'accompagne toujours de la valorisation de l'identité, et donc de l'assujettissement à l'identité. Rappelons tout d'abord que chez Hegel, du moins dans la Phénoménologie de l'esprit, on trouve déjà une théorie de la contribution de la reconnaissance à la domination, mais que rien n'y relie positivement reconnaissance et identité au sens de l'identité individuelle ou collective ${ }^{29}$. Le lien entre reconnaissance et identité ne joue pas davantage de rôle dans «Travail et interaction» de Jürgen Habermas. Axel Honneth associe certes reconnaissance et identité dans La Lutte pour la reconnaissance (ce n'est plus le cas ensuite), mais en se référant à un concept formel d'identité, c'est-à-dire à la forme d'un rapport positif ou négatif à soi, et non aux problématiques de l'identité personnelle, sociale, ou culturelle dont il est question lorsqu'on identifie reconnaissance et assujettissement à l'identité. Puisque Jacques

$26 C f$. A. Honneth, «La reconnaissance comme idéologie».

27 Cf. A. Honneth, La Réification.

28 Voir à ce propos M. Renault, "Le genre de la race: Fanon, lecteur de Beauvoir".

29 Comme le souligne notamment O. Tinland, "Conscience de soi, vie et liberté (chap. 4, introduction et section A)». 
Rancière est l'un de ceux qui, à partir des années 1990, a développé cette critique qui confond Axel Honneth et Charles Taylor, il n'est sans doute pas inutile de rappeler que dans La Parole ouvrière ${ }^{30}$, en 1976, il soulignait lucidement l'importance des enjeux de reconnaissance dans le mouvement ouvrier naissant, tout comme Axel Honneth le fait dans La Lutte pour la reconnaissance ${ }^{31}$. Reconnaissance signifiait alors reconnaissance de l'humanité, comme chez Simone de Beauvoir et Frantz Fanon, et non reconnaissance de l'identité. Le Rancière du milieu des années 1970, encore proche du maö̈sme, aurait-il été moins Français ou plus Allemand que le Rancière de La Mésentente en conférant une fonction positive au concept de reconnaissance? Est-il insignifiant que Rancière ait fini par admettre, dans son débat avec Axel Honneth, qu'il existe bien une "théorie ranciérienne de la reconnaissance "33? Quoi qu'il en soit, de Hegel à Honneth, l'enjeu est bien celui d'une reconnaissance universelle, et non pas de la reconnaissance d'une particularité, et cet enjeu est toujours aussi celui d'une reconnaissance conçue comme un universel concret et non comme universel abstrait. C'est également de cette manière que la reconnaissance est pensée chez Simone de Beauvoir et Frantz Fanon, contre le faux universel que les dominants prétendent incarner.

\section{Mondialisation et institutionnalisation des traditions philosophiques nationales?}

La mondialisation de la philosophie est l'un des phénomènes marquants $\mathrm{du} \mathrm{xx}^{\mathrm{e}}$ siècle. Il existe indéniablement des traditions philosophiques nationales au $\mathrm{XVIII}^{\mathrm{e}}$ siècle, empiriste-lockéenne en Grande-Bretagne, rationaliste-cartésienne en France, idéalisteleibnizienne en Allemagne, bien que l'Europe de cette époque soit également un intense espace de discussions transfrontalières dont les

30 Cf. A. Faure, J. Rancière, La Parole ouvrière.

31 Pour une analyse des évolutions de Rancière concernant la reconnaissance, voir G. Campailla, L'intervento critico di Rancière. Democrazia, riconoscimento, emancipazione ottocentesca.

32 Cf. J. Rancière, La Mésentente.

33 Cf. J.-P. Deranty, K. Genel (dir.), Reconnaissance ou mésentente? Un dialogue critique entre Jacques Rancière et Axel Honneth, op. cit. 
effets ne doivent pas être sous-estimés ${ }^{34}$. L'émergence des nationalismes aux siècles suivants conduisit des penseurs allemands à brocarder la superficialité de la philosophie française, tandis que des Français dénonçaient le totalitarisme de la pensée allemande, mais des «transferts culturels» (par exemple entre philosophie allemande et socialisme français, ou encore entre le kantisme et la Troisième République) continuèrent à produire des effets structurants. Depuis le début $\mathrm{du} \mathrm{xx}^{\mathrm{e}}$ siècle, un processus d'internationalisation de la discussion philosophique s'est ensuite continûment développé, qui a vu notamment le pragmatisme américain influencer la philosophie chinois ${ }^{35}$, avant que la philosophie américaine ne soit colonisée par la philosophie autrichienne, et que la philosophie française ne soit profondément transformée par la philosophie allemande. Le contexte contemporain est marqué par une intensification de cette mondialisation, mais aussi par de puissantes dynamiques de sur-spécialisation des savoirs, y compris en philosophie, qui conduisent notamment à l'institutionnalisation de spécialités nationales profondément anachroniques. L'émergence de spécialisations en "philosophie française» ou en "philosophie allemande" génère et nourrit l'illusion que ces philosophies nationales existeraient indépendamment l'une de l'autre.

Les spécificités de la philosophie française de la deuxième moitié du $\mathrm{Xx}^{\mathrm{e}}$ siècle ne sont pas identifiables indépendamment de la philosophie allemande. Alexandre Kojève, qui n'était ni Français ni Allemand, a impulsé de nouvelles dynamiques à partir d'un commentaire de Hegel inspiré par Marx et Heidegger, Husserl a profondément influencé Foucault et Derrida, etc. Il semble donc qu'il y ait quelque chose d'anachronique à caractériser un phénomène comme la philosophie française d'après-guerre en faisant de son caractère français une caractéristique philosophiquement distinctive, plutôt que géographiquement informative. Cela ne signifie pas que rien de spécifique n'est lié à la catégorie géographiquement informative de «français». Cela signifie seulement que cette spécificité n'est pas celle d'une tradition philosophique, ou d'une école unifiée par un ensemble de principes philosophiques communs. C'est seulement celle de contextes de discussion particuliers dans lesquels se jouent

34 Voir par exemple le cas frappant de l'«invention» de la «conscience»: $c f$. É. BaLIBAR, «L'invention de la conscience».

$35 C f$. E. Renault, "Qui n’a pas fait d'enquête n'a pas droit à la parole?». 
d'une part les effets de la lecture de Hegel par Kojève, qui a pu être exploitée ou critiquée de différentes manières, et d'autre part des phénomènes qui sont pour partie seulement liés de manière polémique à Kojève, et qui contribuèrent au développement conjoint d'une forme d'anti-hégélianisme spécifique, chez Althusser, Foucault, et Deleuze, notamment ${ }^{36}$.

En Allemagne, certes, il y a bien eu à la même époque des écoles philosophiques, et l'une d'entre elles, l'école heideggérienne, a bel et bien prétendu être un phénomène authentiquement allemand, mais le fait que ces écoles aient pu être aussi rivales que l'école heideggérienne et la théorie critique francfortoise ne fournit-il pas une raison supplémentaire de refuser de parler d'«allemand" ou de "germanique» en général? Le signifiant géographique "francfortois" est celui d'une ville et non d'une nation, et il serait pour le moins paradoxal d'en faire un phénomène typiquement allemand: Marx (qui fut notamment le fondateur d'Annales franco-allemandes et vécut la plus grande partie de sa vie en exil) et Freud (un Autrichien qui se forma notamment à Paris et qui mourut exilé) furent-ils spécifiquement Allemands? Aucune de ces deux figures tutélaires de la tradition francfortoise ne l'aurait sans doute admis. Adorno est-il redevenu spécifiquement Allemand après son exil? La question porte en elle sa réponse (surtout si on veut lui éviter de se retourner dans sa tombe). Quant aux générations ultérieures de la théorie critique, qui se sont fortement appuyées sur le pragmatisme américain (comme Jürgen Habermas et Axel Honneth), ainsi que sur les sciences sociales américaines et françaises (Parsons et Durkheim notamment, s'agissant du Honneth du Droit de la liberte $e^{37}$ tout particulièrement), en quoi au juste seraient-elles plus spécifiquement allemandes?

\section{Bibliographie}

Balibar Étienne, "L'invention de la conscience», in: Locke John, Identité et différence, Paris: Seuil, 1998.

36 Voir J.-B. Vuillerod, L'Anti-hégélianisme de la philosophie française des années 1960: constitution et limites d'un renversement philosophique.

37 Cf. A. Honneth, Le Droit de la liberté. 
Bentouhami-Molino Hourya, Races, cultures, identités, Paris: PUF, 2015.

Berner Christian, "La critique de la conscience morale (chapitre 6, section C)», in: Bouton Christophe, Renault Emmanuel (dir.), Lire la Phénoménologie de l'esprit, Paris: ENS Éditions (à paraître).

Butler Judith, Sujets du désir. Réflexions hégéliennes en France au $X X^{e}$ siècle, Paris: PUF, 2011.

Campailla Giovanni, Lintervento critico di Rancière. Democrazia, riconoscimento, emancipazione ottocentesca, Milan: Metelmi, 2019.

Deranty Jean-Philippe, Genel, Katia, (dir.), Reconnaissance ou mésentente? Un dialogue critique entre Jacques Rancière et Axel Honneth, Paris: Éditions de la Sorbonne, 2020.

Faure Alain, Rancière Jacques, La Parole ouvrière, Paris: La Fabrique, 2007.

Fischbach Franck, Hegel, Fichte. La reconnaissance, Paris: PUF, 1999.

FischBACH Franck, “"Lagir de tous et de chacun”: l'autre mot de l'esprit (chapitre 5, sections B et C)", in: Bouton Christophe, Renault Emmanuel (dir.), Lire la Phénoménologie de l'esprit, Paris: ENS Éditions (à paraître).

Habermas Jürgen, La Technique et la Science comme "idéologie», trad. J.-R. Ladmiral, Paris: Gallimard, 1973.

Hegel Georg Wilhelm Friedrich, Phénoménologie de l'esprit, trad. B. Bourgeois, Paris: Vrin, 2018.

Honneth Axel, «La reconnaissance comme idéologie», in: La Société du mépris, Paris: La Découverte, 2006, p. 254-274.

Honneth Axel, La Réification, Paris: Gallimard, 2007.

Honneth Axel, La Lutte pour la reconnaissance, Paris: Gallimard, 2013.

Honneth Axel, Le Droit de la liberté, Paris, Gallimard, 2015.

Honneth Axel, Critique du pouvoir, Paris: La Découverte, 2017.

Honneth Axel, La Reconnaissance. Histoire européenne d'une idée, Paris: Gallimard, 2020.

Jarczyk Gwendoline, Labarrière Pierre-Jean, Les Premiers Combats de la reconnaissance, Paris: Aubier, 1987.

Jarczyk Gwendoline, Labarrière Pierre-Jean, De Kojève à Hegel. 150 ans de pensée hégélienne en France, Paris: Albin Michel, 1996.

Labarrière Pierre-Jean, Structures et mouvement dialectique dans la Phénoménologie de l'esprit de Hegel, Paris: Aubier, 1968.

Marx Karl, «Notes sur James Mill», in: Oeuvres, t. 2: Économie (suite), Paris: Gallimard, 1968. 
Olivier Alain Patrick, «La reconnaissance comme lutte et la reconnaissance comme gratitude: un conflit d'interprétation entre Honneth et Ricœur ", in: Olivier Alain Patrick, Roudaut Maiwenn, Schmidt am Busch Hans-Christoph (dir.), Nouvelles perspectives pour la reconnaissance, Lyon: ENS Éditions, 2019.

Ong-VAn-Cung Kim Sang, "Reconnaissance et vulnérabilité. Honneth et Butler", Archives de philosophie, 2010, t. 73, n 1, p. 119-141. Rancière Jacques, La Mésentente, Paris: Galilée, 1995.

Renault Emmanuel, "Qui n'a pas fait d'enquête n'a pas droit à la parole?», in: Descendre Romain (dir.), Langages, politique, histoire. Avec Jean-Claude Zancarini, Lyon: ENS Éditions, 2015.

Renault Emmanuel, "Reconnaissance et domination chez La Boétie », in: Toto Francesco, Pénigaud de Mourgues Théophile, Renault Emmanuel (dir.), La Reconnaissance avant la reconnaissance. Archéologie d'une problématique moderne, Lyon: ENS Éditions, 2017, p. 27-40.

Renault Emmanuel, Reconnaissance, conflit, domination, Paris: CNRS Éditions, 2017.

Renault Emmanuel, "Travail et interaction. Origine et implications d'une distinction ", in: Aubert Isabelle, Kervégan Jean-François (dir.), Dialogues avec Jürgen Habermas, Paris: CNRS Éditions, 2018, p. 117-128.

Renault Emmanuel, "Théorie de la reconnaissance et négativisme méthodologique", in: Olivier Alain Patrick, Roudaut Maiwenn, Schmidt am Busch Hans-Christoph (dir.), Nouvelles perspectives pour la reconnaissance: Lectures et enquêtes, Lyon : ENS Éditions, 2019.

Renault Matthieu, "Le genre de la race: Fanon, lecteur de Beauvoir", Actuel Marx, n' 55, 2014, p. 36-48.

Ricoeur Paul, Parcours de la reconnaissance. Trois études, Paris: Stock, 2004.

SIEP Ludwig, Anerkennung als Prinzip der praktischen Philosophie: Untersuchungen zu Hegels Jenaer Philosophie des Geistes, Freiburg/ Munich: Albert, 1979.

Siep Ludwig, IкÄHeImo Heikki, Quante Michael (hrsg.), Handbuch Anerkennung, Dordrecht: Springer, 2020, <https://doi. org/10.1007/978-3-658-19561-8_42-1>.

Tinland Olivier, "Conscience de soi, vie et liberté (chap. 4, introduction et section A)», in: Bouton Christophe, Renault Emmanuel (dir.), Lire la Phénoménologie de l'esprit, Paris: ENS Éditions (à paraître). 
Тото Francesco, «L'impensé de Claude-Adrien Helvétius. Le problème de l'estime dans De l'esprit», in: Toto Francesco, Pénigaud De Mourgues Théophile, Renault Emmanuel (dir.), La Reconnaissance avant la reconnaissance. Archéologie d'une problématique moderne, Lyon: ENS Éditions, 2017, p. 167-194.

Vuillerod Jean-Baptiste, L'Anti-hégélianisme de la philosophie française des années 1960: constitution et limites d'un renversement philosophique, thèse soutenue en 2018 à l'Université de Nanterre (une publication partielle est annoncée aux Éditions de l'ENS).

Wildt Andreas, Autonomie und Anerkennung. Hegels Moralitätskritik im Lichte seiner FichteRezeption, Stuttgart: Klett-Cota, 1982.

Ethical Theory and Practice, vol. 16, $\mathrm{n}^{\circ}$ 4, 2013. 